Vol. 8, No. 1, 2021

https://doi.org/10.23939/eem2021.01.001

UDC 330.725.33:330.43

JEL Classification Code C01

\author{
O. Kuzmin \\ Lviv Polytechnic National University, Ukraine, Doctor, Professor, \\ E-mail: Oleh.Y.Kuzmin@1pnu.ua \\ ORCID ID: https://orcid.org/0000-0002-6014-6437 \\ N. Stanasiuk \\ Lviv Polytechnic National University, Ukraine, Doctor, Professor \\ E-mail: Nataliia.S.Stanasiuk@lpnu.ua \\ ORCID ID: https://orcid.org/0000-0002-6885-9431
}

D. Berdnik
Lviv Polytechnic National University, Ukraine, PhD researcher,
E-mail: dmytro.a.berdnik@1pnu.ua

\title{
FORECASTING ECONOMIC RESULT OF MANIPULATION USING GAME THEORY MODELING
}

\begin{abstract}
Manipulations are taking place widely on various capital, commodity, derivative and other markets. They are reported regularly and sometimes causing significant losses. But it doesn't mean that the efforts intended to limit this sort of activity are insignificant. Surveillance budgets, as well as applied fines, are impressing. The annual volume of manipulative attempts and the efforts, intended to deter these attempts, are growing exponentially year after year. The imperfection and low versatility of detection methods are leaving space for successful attempts, making manipulative behavior still attractive. This paper is representing the model, based on the Game Theory and aimed to fit modern requirements of surveillance. The article defines basic problems in manipulation detection and proves model's capability to solve them. However, the problem is reviewed on a general level allowing to elaborate the versatile model, but not a specific manipulative scenario. At the same time, the model allows complementing it with precise tools defining aspects related to actual manipulation. Manipulation and the shaping of it's economic results are reviewed in-depth, revealing it's core phenomenology.
\end{abstract}

Key words: manipulation, model, economic results.

\section{Introduction}

Many commonly known abstractions are very clear on a general level, while their numerical determination or modeling is a huge challenge. Prudence, lawfulness, manipulation, and others are statements frequently used in modern economics and finances. And they are working perfectly for explaining more complex concepts or cases. However, we still missing transparent mathematical or algorithmic tools, defining prudent or imprudent out of commonly available data.

The problem stands even sharper in case if we need to apply deterring against a certain type of phenomena. Besides identification and classification, in this case, we need to prove the intense to define liability. Many domain practitioners, as well as scientists, are able to define clearly what manipulation is. However, we still challenging to detect, classify and prove the manipulative attempt. As will be shown further, technological development brings the problem to a new level.

There are two basic drivers for resolving this problem. First, manipulations are causing significant economic impact on markets and their participants. 


\section{O. Kuzmin, N. Stanasiuk, D. Berdnik}

As we know from regulation reports, it is vast and sometimes catastrophic. Second, the surveillance, related to market abuse and insider compliance regulation, demands significant costly efforts from financial institutions and authorities. Consequently, an average market participant either suffers from manipulation consequences or caries related risk ownership costs.

\section{Problem definition}

As a first approximation, the problem requires a mathematical definition. Such definition should allow analyzing manipulative attempts transparently and allow employing computation facilities. In other words, the analysis should transform from theoretical to mathematical. This paper is called to introduce the model describing manipulation as deliberate strategic interaction. The methods of Game Theory are fitting this goal perfectly.

Many models in economics are intended to describe some aspects on a general level or evaluate these aspects on a rough scale. Meanwhile, the tasks of decision making or deterring are demanding very accurate tools to be utilized. Such tooling demands a deep understanding of phenomena' mechanics. Actually, implied equations should be elaborated from mathematical descriptions of the mechanics of phenomena. Therefore, the paper highlights basic mechanisms of manipulation in their precise definition.

Another aspect of this problem is the universality of demanded tools. The backbone of this model should describe a basic logic, common for all implementations of the phenomena, while variable aspects should be introduced by complementing modules. Certainly, such architecture is possible to be elaborated only from a conscious understanding of manipulation. In other words, our understanding should clearly define common aspects and aspects impured by the context.

Last, but not least, we need to oversee the economic result of phenomena. It is very important for many implementations. As we will define in the following sections, the economic result plays important role in the evaluation as well as in the detection of manipulative intense.

\section{The Overview of available Works}

First, we should start with the state-of-the-art understanding of what manipulation is. As was highlighted in the introduction, the general meaning of manipulation is clear. But as we getting into details, trying to inspect the phenomena deeper, we are facing a versatile set of definitions. It is easy to admit that all of them are defining basically the same phenomena. However, they are focused on different points, recognizing them as root aspects for manipulations. It is reasonable to assume that the majority of these researches are right and the modern regulation should aggregate all these points in a holistic interpretation of phenomena.

It is worth starting from works, analyzing the evolution of phenomena, or bringing a reasonable classification. In accordance with [1] the earliest recognized manipulations took place at Amsterdam Stock Exchange at the beginning of the 17th century. Shortly it became a common practice. Most probably, this information was inherited from [2], also naming Amsterdam as the first recorded venue manipulations took place in. Using materials of Robert Sobel book 'The Big Board', and the Twentieth Century Fund's publication 'The security markets' (1935), investigators elaborated a coherent narration of manipulations' history up to the year of 1992.

Both papers are highlighting the intensifying over the years process of manipulations' development. Attempts, that had been tracked initially, demanded the distribution of fake news or other kinds of misleading information. Later 'bear raids' had tried to impact markets using a supply-demand ratio. Currently, manipulations can be represented by a set of complex cross-market actions, aimed to segregate an impact market and a profit market. Tom C. Lin in his paper [3] gives a detailed comparison of old and new methods of manipulations. Among other differences he highlights the widespread use of electronic tools, impressively facilitating trading as well as manipulation methods.

A deeper look at the problem of manipulations' technical reinforcement was made by Gina-Gail S. Fletcher in her work [4]. It highlights the emerging problem of deterring technically advanced methods of manipulation. The well-known in theory race of technologies between fraudsters and controlling gets feasible shape and able to be 


\section{Forecasting economic result of manipulation using game theory modeling}

studied case-based. Author calls AI and ML as manipulation tools already in use. More precise research of the correlation between technological growth and emerging manipulative activity was made by Douglas Cumming in the work [5]. They sequentially study the development of innovations and the consequent development of manipulative methods.

The classification of manipulative techniques is typically using either an object of manipulation or a method of manipulation to sort manipulations. The good and one of the earliest examples of the first approach can be found in [2]. Here Allen and Gale are giving the following classification:

- Action-based manipulation

- Information-based manipulation

- Trade-based manipulation

In accordance with the authors first two types were clearly outlawed by the Securities Exchange Act of 1934. This document is also dating the first appearance of the modern definition of these manipulations.

This classification is also used by [1]. However, they give a very important generalization, saying that trade-based manipulations are actually aimed to bring misleading information about a real supply-demand ratio to the market. This idea got it's detailed review in the paper [6]. The authors are coming to very interesting results. Their research shows that the stock price may have a function of information exchange between market players. They evidently highlighting that in some circumstances market participants are seeking price signals as one and only sources of information. In such a situation price signal function is not only complementing the information available but shaping it solely. This observation brings us to the conclusion that there is, actually, one type of manipulation, implying misleading trades, direct disinformation, and misleading actions as ways to bring manipulative information to a market.

The classification based on a manipulation method is widely used in the financial area and especially among domain practitioners. Such subdomains, as market abuse regulation, are more focused on the actual ways to manipulate a market, as their core intention is deterring manipulative methods. A wide range of authors is using such classification or focused on it's part. The paper [7] gives a detailed classification of manipulations able to be generalized as insider trading. A deep study on kinds of manipulative methods was made in work [8]. A very resulting attempt to provide an impact-based classification was made in work [9]. The author defines two types of manipulations by the scale of their impact. The first type of manipulations is able to create an allocated risk at a certain instrument. The second type of manipulations is able to create a market-wide systematic risk. In other words, this type of manipulation is able to create financial volatility impacting the whole domain. The interesting aspect is that both types are closely related to the use of misleading information.

However, we need to admit that the most exhaustive classification, based on the method of manipulation, is available in regulators' public materials. FCA, ESMA, SEC, and other international or regional bodies are developing systematic materials intended to define and prevent manipulations of various nature.

Starting the times of Adam Smith economics, as well as finances, become subjects of constant research. Certainly, manipulations became a subject of researchers' attention as well. Following the emerging manipulative strategies, economic science developed it's understanding of manipulations' mechanics and methods. In the last quarter of the 20th-century science came to a more or less modern understanding of manipulations. The overviews of such works can be found in the papers of Allen and Gale [2], Rajesh Aggarwal[10], and others. However, some authors are worth to be highlighted separately, as they are focusing on specific aspects of manipulation.

Oliver Hart in his paper [11] gave some attention to manipulations' modeling, however, considered them as a type of speculation. Later Robert Jarrow [12] introduced his model of manipulations. Considering a wide range of earlier works, including [11], he elaborated an effective model. However, this model is focused on certain types of manipulations and can be scenariodependent. In the work of Rajesh K. Aggarwal [10] the author proposes a model based on a price jump, and able to detect manipulative attempts of a certain kind. The model is used to compare 3 scenarios where different agents are employed. 


\section{O. Kuzmin, N. Stanasiuk, D. Berdnik}

This approach is very effective to highlight the difference appearing in the market where certain manipulation is applied. However, the versatility of this model is under question.

The fundamental assumption that manipulation can be a strategic step, having all attributes like intention, strategy, and expected result, was made by several authors with different levels of approximation. Jules Hedges in his blog post [13] opens a discussion around 'rule braking' as a strategic step in Game Theory. First of all, he quotes a list of scientists, that putting a classic Game Theory under the question from the perspective of limitations, emerging from the phenomena of rules. Rules are considered as common knowledge, and basically shaping the set of strategies players can apply. This attribute of any game implies not only limitations by design but discards the whole concept of rule-breaking. In the author's idea, rule-breaking should be embedded as a natural ability of any player. Certainly, it opens a myriad of discussions around the taxonomy of rulebreaking but makes equilibrium open for nominals representing the rule-breaking.

These ideas found their practical implementation in various works. The investigators used a model based on Game Theory [14] to prove that time-wasting can be a strategic step to win a soccer game. Meanwhile, it is a type of behavior, restricted by international soccer rules. Jean Luc Vila [15] introduced a simple Game Theory model, describing financial market manipulations as a strategic step.

Later, Diana Dezsi introduced her work [16] where another Game Theory model of market manipulation was introduced. This model develops the idea of manipulation as a strategic step. However, the author overrates the role of the National Securities Commission's regulation. It leads to very low attention to other aspects deterring manipulations. However, the idea of market manipulations, as strategic acts, is well proven.

Considering these papers, we can conclude that the Game Theory is an applicable mathematical instrument to be used for manipulations' modeling. Nevertheless, it's toolkit should be complemented by the strategic breaking of rules.
The significant change in manipulations' perception paradigm took place after the introduction of behavioral economics. Even earlier works admitted the role of biases and individuals' behavior. However, the new approach in the understanding of economics made manipulative behavior a core aspect of the phenomena. Chunsheng Zhou and Jianping Mei in their work [1] introduced a manipulation model intended to detect patterns of malicious behavior. But the correlation between a pattern and an intention remains doubtful in this model. This topic is examined in a paper by Cass R. Sunstein [8]. This paper covers many implementations of manipulations in a more casual form, but evidently proves the presence of a behavioral component of manipulation. In the other paper [17] an overview of early statements and basics, elaborated the current understanding of behavioral economics, is given. We can see the history of such core abstractions as bias and manipulation. Among other important aspects, he highlights that some of the widely appearing biases are shaping the tendency of people to be influenced by supposedly irrelevant factors. In his convenience, it brings the foundation for manipulations. The importance of this statement is hard to be overestimated. It turns manipulation from being a kind of 'black magic' to a plain process employing widely known economical processes.

As a keen highlight, the author quotes Amos Tversky joking about his work establishing what was long known to advertisers and used car salesmen. It is important to highlight that Tversky, working on the problematic of decision making, introduced many concepts behavioral economics is based on. This fact, as well as the employment of behavioral economics in modern manipulation analysis, brings us to the conclusion that behavioral aspects should play a key role in state-of-the-art models.

As we can learn from the works of various authors, one of the most important aspects of manipulative processes is information. It can take different ways to obtain manipulation using information, but many authors evidently highlighting the importance of information in manipulative attempts. E. g. the research [18], giving an evaluation of manipulations phenomena from the perspective of information, was made by Jean Luc Vila. 


\section{Forecasting economic result of manipulation using game theory modeling}

However, this and other works of Vila are focused on insider information rather than the general role of information. The paper [10] defines the exceptional role of information in manipulations. He defines a specific role of 'information seekers', who are playing an important role in a fair price formation. He evidently describes a process of manipulation as strongly related to a lack of information in a pool of traders and consequent injection of misleading information. Another important problem highlighted by the author is the detection of possible manipulations which does not occur. This aspect is very important if we want to manage risks o $\mathrm{f}$ manipulation proactively instead of reactive surveillance. Jean-Yves Delort and the colleagues in their paper [19] are evidently proving the concept of misleading information used to manipulate markets. The research, as well as it's core statements, is made on the basis of the vast pool of messages, quoted from the internet message board intended for professional communication of trading individuals. Roland Benabou and Guy Laroque gave a sharp focus on manipulations related to insider information [20].

Last, but not least aspect of manipulations is it's economic result. The most important details of this aspect are described in a paper [9] by GinaGail S. Fletcher. This article provides a general overview of manipulations' impact on financial markets. The author highlights the change of manipulations' understanding that emerged in financial regulation after the crisis of 2008. She claims to review this phenomenon from a new, macroprudential point of view. The article evidently shows that manipulations that appeared in the market are changing the vulnerability landscape, which all market participants are considering. New risks, related to vulnerabilities introduced, are contributing to the risk ownership costs of all market players. This fact brings us to the conclusion, that a very single manipulation raises costs for the entire market. It is important to highlight that understanding of risks, introduced by a manipulation, is essential for an understanding of manipulations' severity.

The problem of manipulations' economic results was examined in a panel of other works. For example, Robert W. Staiger and Alan Sykes [21] highlight that the impact of manipulation on currency exchange markets is hard to be measured. However, the paper estimates a scale of such processes as ten billion. Also, Craig Pirrong [22] examined similar effects on commodity markets.

The pool of mentioned paper shapes requirements to demanded model.

- The model should consider manipulation not as an untypical phenomenon, but as a strategic step of a market agent.

- The model should explain the behavioral phenomena of manipulation participants.

- The model should imply a conscious explanation of the information exchange processes taking place during a manipulation.

- The model should outline the economic result of manipulation for all parties.

The listed requirements are coherent with the problem definition given earlier.

\section{Model}

The requirement of versatility makes us define the model on a very high level of abstraction. It should lead us to the understanding of manipulation's essence, but not a given interpretation. Therefore, our model will review the game example, defining the basics of the process without deep diving in exchange details. The examination of all available manipulative scenarios will take plenty of time and should be a subject of a separate article.

Let's assume we have a card deck of 3 cards. There are Ace, King, and Jack with the traditional hierarchy. After a deck is shuffled, each player gets one card to play. The remaining card is unknown to both players. Players are giving a minimal bet. Players (starting player one, further in turns) should call on of following options:

- Fall - player considers himself a losing party and refuses to play this round (losing his bet).

- Check - player suggests checking cards without raising a bet.

- Raise - player suggests raising bets. Let's assume it's ' $k$ ' times bigger than initial bet.

Getting the last proposition another player should either support a new bet or fall. It worth mentioning the additional rule able to sound naive or too abstract, however, it's going to play important role in our research. Players should behave honestly. 
We can describe this game in Game Theory equations. Thus, we have a game:

$$
\begin{aligned}
& N=\left\{p_{1}, p_{2}\right\}, \\
& S=\left\{S_{1}, S_{2}\right\} .
\end{aligned}
$$

In a step 0 each player gets his card. Let's call it a Nature's step and conclude that it has 6 combinations in total. Fig. 1 represents all possible strategies, coming out of any Nature's step as a tree-diagram. They are identical for each of step 0 combinations and creating 42 possible strategies with consequent outcomes. The sum of outcomes equal to 0 for each of players, confirms the exhaustiveness of this set of strategies (Fig. 1).

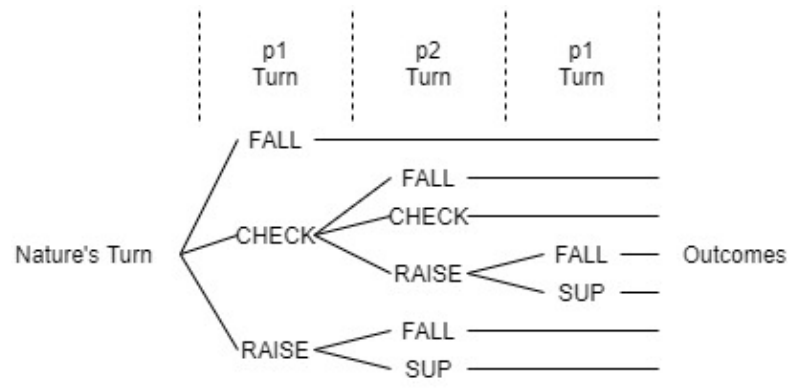

Fig. 1. Tree-diagram of possible strategies

Obviously, each of these 42 strategies consists of standard actions defined by the rules of the game. Let's bring each of actions a referring index (tabl. 1).

\begin{tabular}{|c|c|c|}
\hline Index & Action & Description \\
\hline $\begin{array}{c}(\boldsymbol{A} ; \boldsymbol{K}),(\boldsymbol{A} ; \boldsymbol{J}) \\
(\boldsymbol{K} ; \boldsymbol{A}) \\
(\boldsymbol{K} ; \boldsymbol{J}),(\boldsymbol{J} ; \boldsymbol{A}) \\
\quad(\boldsymbol{J} ; \boldsymbol{K})\end{array}$ & $\begin{array}{l}\text { Nature } \\
\text { turns' } \\
\text { outcome }\end{array}$ & $\begin{array}{l}\text { Any strategy starts with the outcome of nature's turn, } \\
\text { where players are getting mentioned cards accordingly. }\end{array}$ \\
\hline$F_{1}, F_{2}$ & Fall & $\begin{array}{l}\text { Player considers himself a losing party and refuses to play this round (losing his } \\
\text { bet). Can be applied at any stage. }\end{array}$ \\
\hline$C_{1}, C_{2}$ & Check & Player suggests checking cards without raising a bet. \\
\hline$R_{1}, R_{2}$ & Raise & Player suggests raising bets \\
\hline$S_{1}, S_{2}$ & Support & Support a new bet. \\
\hline
\end{tabular}

Expected actions in accordance to possible strategies

The consequent set of array descriptions can be defined as:

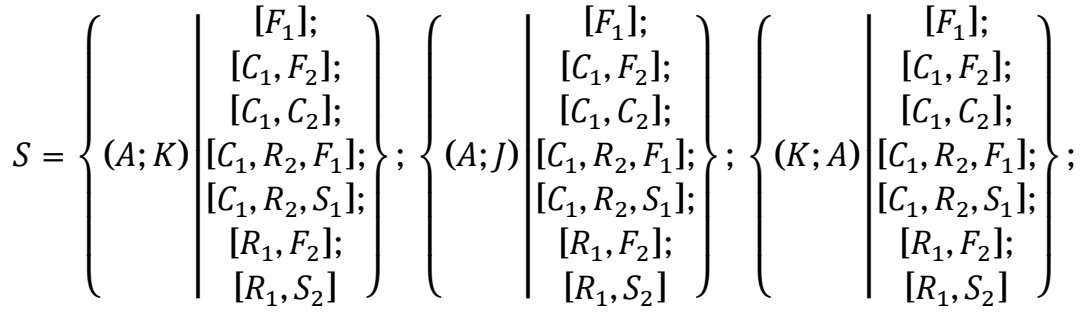

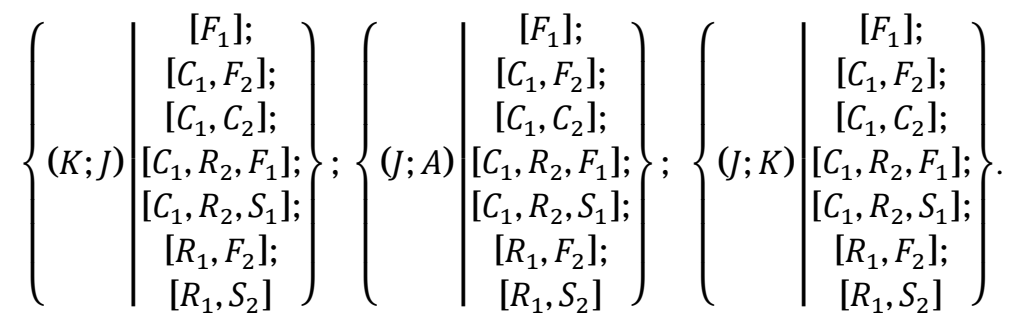

However, from the very first glance, we can spot several strongly dominated strategies. Consequently, the tree-diagram, as well as array representation are able to be shorted using the Carmell rule as follows:

$$
\begin{gathered}
(A ; K) \\
(A ; J)
\end{gathered} \Rightarrow U_{1}\left(s\left[R_{1}, . .\right]\right)>U_{1}\left(s\left[F_{1}\right]\right) ;
$$

$$
\begin{gathered}
(A ; K) \\
(A ; J)
\end{gathered} \begin{gathered}
>U_{1}\left(s\left[C_{1}, R_{2}, S_{1}\right]\right)> \\
\quad>U_{1}\left(s\left[C_{1}, R_{2}, F_{1}\right]\right) ; \\
(K ; A) \\
(J ; A) \\
\stackrel{(K ; A)}{(J ; A)} \Rightarrow U_{2}\left(s\left[R_{1}, S_{2}\right]\right)>U_{2}\left(s\left[R_{1}, F_{2}\right]\right) ; \\
\Rightarrow U_{2}\left(s\left[C_{1}, C_{2}\right]\right)>U_{2}\left(s\left[C_{1}, F_{2}\right]\right) ;
\end{gathered}
$$




$$
\begin{gathered}
(A ; J) \\
\stackrel{(K ; J)}{(J ; A)} \Rightarrow U_{2}\left(s\left[R_{1}, F_{2}\right]\right)>U_{2}\left(s\left[R_{1}, S_{2}\right]\right) ; \\
\stackrel{(J ; K)}{ } \Rightarrow U_{1}\left(s\left[C_{1}, R_{2}, F_{1}\right]\right)> \\
\quad>U_{1}\left(s\left[C_{1}, R_{2}, S_{1}\right]\right) .
\end{gathered}
$$

Following strategies are able to be dismissed as weakly dominated:

$$
\begin{aligned}
& \underset{(K ; J)}{(K ; A)} \Rightarrow U_{1}\left(s\left[C_{1}, . .\right]\right) \geq U_{1}\left(s\left[F_{1}\right]\right) ; \\
& \underset{(J ; K)}{(A ; K)} \Rightarrow U_{2}\left(s\left[C_{1}, C_{2}\right]\right) \geq U_{2}\left(s\left[C_{1}, F_{2}\right]\right) .
\end{aligned}
$$

This stage is very important. Dominations defined in (4)-(11) are agnostic to further conclusions as well as to honesty rule, mentioned during the game definition. We are going to return to this highlight in some of further stages.

Being rational, and obeying rules, $p_{1}$ will not apply $\left[R_{1}, \ldots\right]$ strategy if he got Jack. The expectation of $\left[R_{1}, S_{2}\right]$ in this case will be irrational, while the expectation of $\left[R_{1}, F_{2}\right]$ will contradict with the honesty rule. Consequently, whole branch $\left[R_{1}, \ldots\right]$ is strongly dominated. And symmetrically, $p_{2}$, being rational, and obeying rules, will not apply $\left[C_{1}, R_{2}, \ldots\right]$ strategy if he got Jack. Moreover, assuming that the value of time for both of players is not equal to zero, the most rational strategy having Jack is to fall as soon as possible. Because any other actions are not able to bring any better result without breaking the rule of honesty. However, they will always utilize larger or the same amount of time. Assuming that each turn of trades takes $\tau$ amount of time, we can come to following conclusion:

$$
(J ; \ldots) \Rightarrow U_{1}\left(s\left[F_{1}\right]\right)=-1-f(\tau) .
$$

While any other branch, having more turns of trade, will lead to lower outcome. Therefore, for player 1 , having Jack, any strategy other than falling from the very beginning are strongly dominated.

$$
\begin{aligned}
& (J ; A) \\
& (J ; K)
\end{aligned} \Rightarrow U_{1}\left(s\left[F_{1}\right]\right)>U_{1}\left(\forall s \neq s\left[F_{1}\right]\right) .
$$

On the other hand, having Ace, player 1 will always try to maximize his outcome and therefore applies the shortest strategy able to give biggest outcome.

$$
\underset{(A ; K)}{(A ; J)} \Rightarrow U_{1}\left(s\left[R_{1}, \ldots\right]\right)>U_{1}\left(\forall s \neq s\left[R_{1}, \ldots\right]\right) \text {. }
$$

It is interesting to admit that 13 and 14 , being a part of common knowledge about the game, are taking a role of signals between players. This aspect is extremely important. In situations when one and only strategy can be performed by a prudent player, the recognition of this strategy by other players leads to the identification of this situation without being actually disclosed. As was discovered in [6] on the example of stock quotations, and as it obviously recognized in a given model, strategic actions of players in certain conditions may have a signal function. Consequently, this function should be recognized where needed and taken into consideration.

Knowing that $s\left[R_{1}, \ldots\right]$ is rational only in case if $p_{1}$ got Ace, $p_{2}$ will apply $s\left[R_{1}, F_{2}\right]$ as one and only reasonable strategy. Consequently, having King, $p_{1}$ will apply $\left[C_{1}, \ldots\right]$, demanding explicit actions from $p_{2}$. Any other strategy in this case will break either honesty or rationality.

Out of the context of information exchange, conducted by signal functions of strategies, previous conclusions may seem irrational. Evaluating this game from the perspective of bare Game theory, we can face the situation when we are unable to discard dominated strategies any longer, but the real players are easily shrinking to a single strategy. It leads us to a very important conclusion, that consideration of information exchange makes prudent behavior able to be modeled where it was impossible earlier.

Finally, (3) is collapsing to:

$$
\begin{gathered}
\left\{(A ; K) \mid\left[R_{1}, F_{2}\right]\right\} ; \\
\left\{(A ; J) \mid\left[R_{1}, F_{2}\right]\right\} ; \\
\left.S(K ; A) \mid\left[C_{1}, R_{2}, F_{1}\right]\right\} ; \\
\left\{(K ; J) \mid\left[C_{1}, F_{2}\right]\right\} ; \\
\left\{(J ; A) \mid\left[F_{1}\right]\right\} ; \\
\left\{(J ; K) \mid\left[F_{1}\right]\right\} .
\end{gathered}
$$

We need to summaries few important results.

- For each Nature's step there is one and only dominating strategy.

- (15) is the Nash equilibrium.

- The probability of each strategy is equal to probability of referring Nature's step $\left(\sigma=\frac{1}{6}\right)$.

- The sum of outcomes for each player is equal to zero.

$$
\sum_{i=1} U_{1, i}=\sum_{i=1} U_{2, i}=0
$$

- The sum of outcomes for each player weighted by their probabilities is equal to zero.

$$
\sum_{i=1} U_{1, i} \cdot \sigma_{1, i}=\sum_{i=1} U_{2, i} \cdot \sigma_{2, i}=0 .
$$




\section{O. Kuzmin, N. Stanasiuk, D. Berdnik}

- In $2 / 3$ of rounds a taken player clearly understands his expected outcome. The remaining $1 / 3$ of rounds represents uncertainty with the risk of minimal bet.

Let's briefly review the same game excluding the honesty rule. However, let's highlight that it is absent for both players and both are aware of it's absence.

$$
\begin{aligned}
& N^{U}=\left\{p_{1}, p_{2}\right\}, \\
& S^{U}=\left\{S_{1}^{U}, S_{2}^{U}\right\} .
\end{aligned}
$$

The expressions (4)-(11) are going to remain valid, as their definition not uses the honesty rule. Remaining strategies can be examined using Bayesian games equilibriums or by introducing values of risks for both players, as factors impacting outcomes for 'unfair' strategies. This game scenario completely correlates with the conclusions of [9]. Both players are going to own additional risks related to the threat of the non-honest game. From the perspective of strategies' set, players will not dismiss $\left[R_{1}, \ldots\right]$ and $\left[C_{1}, R_{2}, \ldots\right]$ as dominated due to the risk of the unfair game. So during a share of rounds, their risks will be $\mathrm{k}$ times more.

However, both approaches are going to lead to use of $\sigma=\frac{1}{6}$ as a core argument defining Bayesian probabilities as well as values of risks. Consequently, $p_{1}$ and $p_{2}$ are going to form $S^{U}>S$ (meaning number of strategies bigger than represented in (15)). The final set of strategies of $S^{U}$ is not important for us. We need only the understanding that, this game will have the following properties:

$$
S^{U}=\left\{S_{1}^{U}, S_{2}^{U}\right\} .
$$

- $S^{U}$ is the Nash equilibrium in mixed strategies.

- The probability of each strategy derivates to probability of referring Nature's step $\left(\sigma=\frac{1}{6}\right)$.

- The sum of outcomes for each player is equal to zero.

$$
\sum_{i=1} U_{1, i}^{U}=\sum_{i=1} U_{2, i}^{U}=0
$$

- The sum of outcomes for each player weighted by their probabilities is equal to zero.

$$
\sum_{i=1} U_{1, i}^{U} \cdot \sigma_{1, i}=\sum_{i=1} U_{2, i}^{U} \cdot \sigma_{2, i}=0 .
$$

- Only in $1 / 3$ of rounds a taken player clearly understands his expected outcome (he has an Ace). The remaining $2 / 3$ of rounds represents uncertainty with the risk of minimal bet $\mathrm{k}$ times.

As will be explicitly shown further, (22) is very important. It shows that the end results of both games are equal in a long run. It is important to understand that honest game and mutual ignoring of honesty leads to the very same outcome in statistically significant series of games. Also, the last point represents statements of [9]. We may conclude that (18)-(22) represent a market condition after the very first manipulation was conducted.

For the reviewing the core of this paper, let's assume that $p_{1}$ and $p_{2}$ are running the game defined in (1) and (2). However, the $p_{2}$ decided to break the honesty rule solely. Earlier we identified that strategies (15) are able to act as signals between players. Consequently, in the situation $(K ; \ldots) p_{1}$ will apply $s\left[C_{1}, \ldots\right]$, flagging to $p_{2}$ his strategic position. In a case of honest game $p_{2}$ should apply $s\left[C_{1}, R_{2}, \ldots\right]$ or $s\left[C_{1}, F_{2}\right]$ depending on a card he has. And what is important, his action will be a signal to $p_{1}$ as well. Therefore, having King and being rational, $p_{1}$ will never apply $s\left[C_{1}, R_{2}, S_{1}\right]$ as he believes that $p_{2}$ got Ace.

Considering this signal interaction $p_{2}$ will apply manipulation, trying to convince $p_{1}$ that he is in a different game situation than he actually is. He will not apply any other strategies different from $S_{2}$ to not discover himself being not honest. Consequently, the game will not transform into form, defined in (18), (19). Let's define this game formally to examine the difference with (1) and (18).

$$
\begin{aligned}
& N^{M}=\left\{p_{1}, p_{2}\right\}, \\
& S^{M}=\left\{S_{1}, S_{2}^{M}\right\} .
\end{aligned}
$$

Where $S_{1}$ is identical to (2) and $S_{2}^{M}$ can be defined as:

$$
S_{2}^{M}=S_{2}-s\left[C_{1}, F_{2}\right]+s[C_{1}, \overbrace{M_{2}\left\langle R_{2}\right\rangle}^{(K J) \rightarrow(K, A)}, \ldots] .
$$

$$
\text { Where } \overbrace{M_{2}\left\langle R_{2}\right\rangle}^{(K, J) \rightarrow(K, A)} \text {, is the manipulation }
$$
performed by $p_{2}$ intended to convince $p_{1}$ that he is in a game position $(K, A)$ but not $(K, J)$, as actually is. Certainly, such a strategy is strongly dominating the honest strategy. 
Forecasting economic result of manipulation using game theory modeling

$$
\begin{aligned}
(K ; J) \Rightarrow U_{2} & \left(s[C_{1}, \overbrace{M_{2}\left\langle R_{2}\right\rangle}^{(K J) \rightarrow(K, A)}, \ldots]\right)> \\
& >U_{2}\left(s\left[C_{1}, F_{2}\right]\right) .
\end{aligned}
$$

Being rational and manipulated $p_{1}$ will apply strategy $s\left[C_{1}, R_{2}, F_{1}\right]$. As far as behavior of $p_{1}$ gives him a signal that he is in game situation of $(K, A)$, the strategy $\left[C_{1}, R_{2}, S_{1}\right]$ will never take place, being strongly dominated.

Consequently, (15) will transform to:

$$
\begin{aligned}
& \left\{(A ; K) \mid\left[R_{1}, F_{2}\right]\right\} ; \\
& \left\{(A ; J) \mid\left[R_{1}, F_{2}\right]\right\} ; \\
& \left\{(K ; A) \mid\left[C_{1}, R_{2}, F_{1}\right]\right\} ; \\
& S^{M}=\left\{(K ; J) \mid[C_{1}, \overbrace{M_{2}\left\langle R_{2}\right\rangle}^{(K J) \rightarrow(K, A)}, F_{1}]\right\} ; \\
& \left\{(J ; A) \mid\left[F_{1}\right]\right\} ; \\
& \left\{(J ; K) \mid\left[F_{1}\right]\right\} .
\end{aligned}
$$
results.

And as in the previous models let's list

- For each Nature's step there is one and only dominating strategy.

- (27) is the Nash equilibrium. Here we need to highlight that the status of Nash equilibrium is applicable only in case if $p_{1}$ is manipulated and the fact of single or repetitive manipulation is not discovered.

- The probability of each strategy is equal to probability of referring Nature's step $\left(\sigma=\frac{1}{6}\right)$.

- The sum of outcomes for player two is bigger than the sum of outcomes for player one.

$$
\sum_{i=1} U_{2, i}>\sum_{i=1} U_{1, i}
$$

The precise value of difference can be defined as:

$$
\begin{aligned}
& \sum_{i=1} U_{2, i}-\sum_{i=1} U_{1, i}= \\
= & 2 U_{2}\left(s[C_{1}, \overbrace{M_{2}\left\langle R_{2}\right\rangle}^{(K,) \rightarrow(K, A)}, F_{1}]\right) .
\end{aligned}
$$

- The sum of outcomes for player two weighted by their probabilities is bigger than the sum of outcomes weighted by their probabilities for player one.

$$
\sum_{i=1} U_{2, i} \cdot \sigma_{2, i}>\sum_{i=1} U_{1, i} \cdot \sigma_{1, i} .
$$

The precise value of difference can be defined as:

$$
\begin{aligned}
& \sum_{i=1} U_{2, i} \cdot \sigma_{2, i}-\sum_{i=1} U_{1, i} \cdot \sigma_{1, i}= \\
& =2 U_{2}\left(s\left[C_{\text {Analysis }}^{\left[C_{M_{2}\left\langle R_{2}\right\rangle}^{(K, J) \rightarrow(K, A)}, F_{1}\right.}\right]\right) \cdot \sigma_{2, i} .
\end{aligned}
$$

The example of three card poker evidently showed that the proposed model is capable to analyze strategic interactions employing manipulation. For elaborating more general regularities and providing analysis of results gained, we need to generalize proposed method. Being more abstract, it can be applied for various strategic interactions. Accordingly, the game should be defined as:

$$
\begin{aligned}
N & =\left\{p_{1}, \ldots, p_{n}\right\}, \\
S & =\left\{S_{1}, \ldots, S_{n}\right\},
\end{aligned}
$$

Let's assume that this game has Nash equilibrium

$$
\overline{\bar{S}} \in S \Rightarrow \overline{\bar{S}}=\left\{\overline{\overline{S_{1}}}, \ldots, \overline{\overline{S_{n}}}\right\},
$$

It has no difference rather it is in mixed strategies or not. The core aspect is that any of $p_{1}-p_{n}$ are not going to apply any better strategy, what is practically the definition of Nash equilibrium. The same aspect is important for $\overline{\bar{S}}$ in mixed strategies.

As abstract game could have any sum, but not only a zero sum, the sum of outcomes for each player is equal to certain value.

$$
\sum_{i=1} U_{1, i}=\text { const }_{1}, \ldots, \sum_{i=1} U_{n, i}=\text { const }_{n} .
$$

Accordingly, the sum of outcomes for each player weighted by their probabilities is equal to certain constants as well.

$$
\sum_{i=1} U_{1, i} \cdot \sigma_{1, i}=\text { const }_{1}, \ldots, \sum_{i=1} U_{n, i} \cdot \sigma_{n, i}=\text { const }_{n}(
$$

Where actual values of $U$ can be defined with various formulas in accordance with a precise case of strategic interaction.

Let's assume that $p_{n}$ has a strategy $s[\ldots, X, \ldots]$. And we can state that it is a dominating strategy in condition $(B)$, but not in any other condition, so:

$$
\begin{gathered}
(B) \Rightarrow s[\ldots, X, \ldots] \in S, s[\ldots, X, \ldots] \in \overline{\bar{S}}, \\
(\forall \neq B) \Rightarrow s[\ldots, X, \ldots] \in S, s[\ldots, X, \ldots] \notin \overline{\bar{S}} .
\end{gathered}
$$




\section{O. Kuzmin, N. Stanasiuk, D. Berdnik}

Consequently, if it is possible that actions of $p_{n}$ are having an effect of signal to other players, we can assume a game:

$$
\begin{gathered}
N^{M}=\left\{p_{1}, \ldots, p_{n}\right\}, \\
S^{M}=\left\{S_{1}, \ldots, S_{n}\right\},
\end{gathered}
$$

With referring Nash equilibrium:

$$
\overline{\overline{S^{M}}} \in S^{M} \Rightarrow \overline{\overline{S^{M}}}=\left\{\overline{\overline{S_{1}}}, \ldots, \overline{\overline{S_{n}^{M}}}\right\},
$$

Where some of the strategies is replaced with the manipulative strategy

$$
s[\ldots, \stackrel{\forall \neq B \rightarrow B}{M\langle X\rangle}, \ldots] \in S^{M} .
$$

(26) is very important to understand the motivation, pushing $p_{2}$ to manipulation. The manipulation brings value in this strategy. In a generalized view (26) will appear as:

$$
\begin{aligned}
(\forall \neq B) & \Rightarrow U_{n}(s[\ldots, \overbrace{M\langle X\rangle}^{\forall \neq B \rightarrow B}, \ldots])> \\
& >U_{n}(s[\ldots, Z, \ldots]) .
\end{aligned}
$$

As explicitly shown in (31), $p_{2}$ has strategic advantage by applying the manipulative strategy. Manipulation is risky and depends on many factors. Any intentional participant of manipulation must have a practical reason to perform it. Consequently, in practical tasks we need to identify an outcome of honest game in observable period versus an outcome of manipulation. In generalized view (30) will transform to:

$$
\sum_{i=1} U_{n, i}^{M} \cdot \sigma_{n, i}>\sum_{i=1} U_{n, i} \cdot \sigma_{n, i} \cdot
$$

Even not knowing the outcome of manipulation (f. e. in case we don't know a manipulative strategy), we can spot a deviation from expected outcome. The aspect of manipulation's profitability was highlighted in [4] one of the core factors in recognition of manipulative attempt. The same idea was introduced by [19].

It is very easy to observe that in a long run the strategy $s[\ldots, X, \ldots]$ will appear with different probabilities for $N$ and $N^{M}$. As shown in (37) (38), it is result of manipulation, but the statistical deviation is able to be detected by not acknowledged observer. And symmetrically, the probability of $s[\ldots, Z, \ldots]$ in $N$ and $N^{M}$ will be different. This factor is very important as sum of strategies represents the behavioral preferences of each player. Analyzing these preferences, we can identify a type of a game we are observing. As was highlighted in [17] and
[1], the behavioral approach to understanding of manipulation is very promising.

Another important outcome is that $s[C_{1}, \overbrace{M_{2}\left\langle R_{2}\right\rangle}^{(K, J) \rightarrow(K, A)}, \ldots]$ is generally described by (3) as $\left\{(K ; J) \mid\left[C_{1}, R_{2}, \ldots\right]\right\}$. Considered strongly dominated (8), it was excluded out of $S$ and we can not find it in (15). It means that for any given game the manipulative action is technically possible and an initial list of strategies should contain this strategy, as it shown in (37). However, this strategy is typically strongly dominated without a manipulative component, as respectively shown in (38). This idea becomes more understandable if we will imagine that we, as an observer, can see only a card of $p_{2}$. In such case, we can not evaluate a game in general, but we can spot rational and irrational steps. Practically, having Jack $p_{2}$ should not raise a bet. Consequently, in a given conditions the use of strategy $\left\{(K ; J) \mid\left[C_{1}, R_{2}, \ldots\right]\right\}$ implies manipulative intense. In a more general meaning actions, being not coherent with available disposition, are most likely caused by manipulative attempt. It can be observed basing on example of (3) and (15), where any actions, listed in (3) other than listed in (15) are not adequate in application to a given conditions.

It also brings us to the problem first mentioned in [10]. The problem highlighted by the author is the detection of possible manipulations, which do not occur. This aspect is very important if we want to manage risks of manipulation proactively instead of reactive surveillance. From this perspective, a dominated strategy with a potential signal function can be considered as potential manipulation. Consequently, any opportunist, stepping in such a position, can be recognized before the effect on a market.

This situation can be revised from an alternative angle. As was indicated in [19] and the list of other works, uncertainty plays a strong role in setting up manipulation and it's actual effect. Therefore, market authorities can deter manipulative opportunities, detected as described above, by disclosing market evaluation information. Being able to aggregate market data and having unquestionable authority, market regulation bodies can compensate an information vacuum eliminating manipulative opportunities. 


\section{Economic Result of Manipulation}

The economic effect of manipulation is studied in a works $([21,22])$. The common idea of marketwide losses strongly related to the appearance of new risks at a given market. After the very first manipulation, all market participants are starting o own a risk to be manipulated. The ownership cost for this risk will depend on a size of possible manipulation, it's probability, and costs of measures aimed to deter this risk. The final expression for these costs is very case-based and should be elaborated on by risk management specialists. For the matter of this study, we can define it as a function $f(R)$.
This process has it's reversal effect. The emergence of related risks pushes a share of market players to lower their activity or to exit a market at all. This and other aspects, changing the supplydemand ratio, are able to lower the asset's price for a sufficient period. In such circumstances, all asset holders are incurring losses proportional to a price drop. It can be defined as $\left(P_{A}^{1}-P_{A}^{2}\right) V_{A}$, where $V_{A}$ is a volume of affected assets. As a basic market can affect related derivatives, we need to apply the same ratio to its derivatives. Summing up the outcomes above, we can come to the expression defining market's economic effect as follows.

$$
E_{\text {market }}=N_{\text {market }} f(R)+\left(P_{A}^{1}-P_{A}^{2}\right) V_{A}+\sum_{j=1}^{d}\left(P_{j}^{1}-P_{j}^{2}\right) V_{j} .
$$

Where $N_{\text {market }}$ is a number of active market players (actually the pool of participants who considers the risk $f(R)), \mathrm{P}$ is a price of an asset or it's derivative, $\mathrm{V}$ is a volume of a given market or a market of it's derivative, $d$ is a number of affected derivatives.

Works [21], [22] and other authors (f. e. [19]) are highlighting the personal profit as a main driver of malicious behavior. (26) and (43) are explicitly showing this phenomenon. Therefore, these equations can be taken as a basis for defining of manipulators economic result.

However, we need to keep in mind that in some conditions a player, who's tried to be manipulated, can ignore a signal function and apply in response a strategy breaking the course of manipulation. In this case, with a probability $\gamma_{X}$ of such an event, he will incur losses (the outcome of normally dominated strategy). This aspect has a bit recursive nature but introduces one very important

aspect of real implementations. In our early assumptions, we took that a player, accepting a signal function, is solidly leaded by rationality and selects one and only strategy. However, in practice, he will have rather a panel of strategies, implying a strategy to ignore a signal function. Again, this concept may seam recursive, but it still operates in an initial set of strategies (before applying the Carmel rule). Most probably, in a real application, we will clearly see a concurrent strategy, which will be even more common for Nesh equilibrium in mixed strategies.

In addition, if the market is regulated, his activity may draw a regulator's attention, causing fines and prosecution. As we can learn from the practice of modern regulators, such impact will depend on the manipulation scale and other parameters. Let's assume it as a function of $E_{\text {market }}$ with a probability of $\rho_{\text {Reg }}$.

$$
E_{M}=U_{M}(s[\ldots, \stackrel{\forall \neq B \rightarrow B}{\forall\langle X\rangle}, \ldots]) \cdot \sigma_{M}+U_{X}(s[\ldots, \stackrel{\forall \neq B}{\widetilde{X}}, \ldots]) \cdot \gamma_{X}+f\left(E_{\text {market }}\right) \cdot \rho_{\text {Reg }} .
$$

The $f\left(E_{\text {market }}\right) \cdot \rho_{\text {Reg }}$ component may appear artificial. However, if we will consider manipulation itself, as a subgame in a bigger game, this new game will perfectly match the game described by Diana Dezsi in her work [16], where results of regulators' actions are shaping the outcome of a manipulator's strategy.

Despite some uncovered operational losses, this equation describes the outcome of manipulation. It is important to admit that it appears naturally

from the outcome of referring strategy. Other components are appearing from alternative strategies or from a higher-scale game.

\section{Conclusions and perspectives of further studies}

A list of works mentioned here already are evidently proving the potential of Game Theory for modeling interactions assuming an opportunity for manipulation. The proposed model uses this 


\section{O. Kuzmin, N. Stanasiuk, D. Berdnik}

potential completely, proposing an efficient and versatile mathematical mechanism. The model defines a high-level canvas explaining the mechanics of manipulation, while the precise market processes can be embedded in the model to define outcomes of strategies. It allows understanding a manipulation concept-wise but analyzing a precise case employing a deep understanding of it's mechanisms and numeric outcomes.

The model considers manipulation as a strategic step. This approach leads to the identification of opportunistic nature of manipulations. It appears due to the fact that manipulation, evaluated as a strategy, demands a prudential basis for it's implementation and it's dominance to be selected. The malicious prudentiality of a manipulator gets it's definition and explanation. This is a key step to interpret malicious behavior.

Another important behavioral aspect is introduced by a wide utilization of signal function in the proposed model. The many of earlier quoted articles are identifying the importance of information in market operations and in the implementation of manipulative attempts. In the given model the signal function of a certain behavior is effectively embedded in strategic interaction.

Last but not the least, the model elaborates conscious equilibriums of economic results for the market as well as for manipulating individual.

Further development of this model can expand in two main directions. Practical use of this model demands a toolkit of equations, introducing outcomes of market activities to the model. In the perfect case, this toolkit should be optimized for program implementation, considering modern volumes of trades. Second, the risk component of market's economic result of manipulation demands a special study, proposing effective numerical methods. These numeric methods are important in the evaluation of manipulation severity, being a cornerstone in regulation.

\section{References}

1. Chunsheng, Z., \& Jianping, M. (2003). Behavior Based Manipulation. NYU Working Paper No. FIN-03-028.
2. Franklin, A. \& Gale, D. (1992). Stock-Price Manipulation. The Review of Financial Studies, 5 (3), 503-529.

3. Lin, T. C. (2017). The New Market Manipulation. Emory Law Journal Vol. 66. 1253.

4. Gina-Gail S., F. (2021). Deterring Algorithmic Manipulation. Vanderbilt Law Review, 74 (2).

5. Cumming, D. \& Ji, Sh. \& Peter, R. \& Tarsalewska, M. (2020). Market manipulation and innovation, Journal of Banking \& Finance, Elsevier, 120(C).

6. Goldstein, I., \& Guembel, A. (2002). Manipulation, the allocational role of prices and production externalities, OFRC Working Papers Series, Oxford Financial Research Centre.

7. Butler, R. S. R. LLP (2007). Market Abuse and the Commodities Markets, Client Alert 07-029,

London. Available at: reedsmith.com/-/medial files/perspectives/2007/04/market-abuse-and-thecommodities-market/files/market-abuse-and-thecommodities-market/fileattachment/market_abuse_ etc_client_alert.pdf

8. Cass, R. S. (2016). Fifty Shades of Manipulation, Journal of Marketing Behavior 1 (3-4), 213-244. http://dx.doi.org/10.1561/107.00000014

9. Gina-Gail, S. F. (2020). Macroeconomic Consequences of Market Manipulation, 83 Law and Contemporary Problems, 123-140. Available at: https://scholarship. law.duke.edu/lcp/vol83/iss1/8

10. Aggarwal, R. K. \& Wu, G., (2003). Stock Market Manipulation - Theory and Evidence (March 11, 2003). AFA 2004 San Diego Meetings

11. Hart, O. D. (1977). On the Profitability of Speculation, Quarterly Journal of Economics, 90, 579-596.

12. Jarrow, R. A. (1992). Market Manipulation, Bubbles Corners and Short Squeezes, Journal of Financial and Quantitive Analysis, 27 (3), 311 336.

13. Hedges, J. (2017). Breaking the rules, Available at: julesh.com/2017/08/16/breaking-the-rules/

14. Greve, H. R., Rudi, N. \& Walvekar, A. (2019). Strategic rule breaking: Time wasting to win soccer games. Available at: https://doi.org/ 10.1371/journal.pone.0224150

15. Jean-Luc Vila, J. L. (1989). Simple games of market manipulation, Economics Letters, 29 (1), 21-26.

16. Dezsi, D. A (2011). Game Theory Model Of Stock Exchange Arket Manipulation, International Conference Of Scientific Paper Afases 2011, Brasov, 26-28 May 2011 
Forecasting economic result of manipulation using game theory modeling

17. Sunstein C. R., (2018). The rise of behavioral economics: Richard Thaler's misbehaving, Journal of Behavioral Economics for Policy, Society for the Advancement of Behavioral Economics (SABE), 2(1), 53-57.

18. Vila, J. L., (1987). The Role of Information in the Manipulation of Futures Markets, University of Pennsylvania, Center for Analytic Research in Economics and the Social Sciences, 54.

Delort, J.-Y. et al. (2021).. The Impact of Manipulation in Internet Stock Message Boards. International Journal of Banking and Finance, 8 (4), 1-18.
20. Benabou, R. \& Laroque, G. (1992). Using Privileged Information to Manipulate Markets: Insiders, Gurus, and Credibility, The Quarterly Journal of Economics, 107 (3), 921-958.

21. Staiger, R. W. and Sykes, Al. (2008). 'Currency Manipulation' and World Trade, Stanford Law and Economics Olin Working Paper No. 363, Rock Center for Corporate Governance Working Paper No. 43.

22. Pirrong, Cr., (2017). The economics of commodity market manipulation: A survey, Journal of Commodity Markets, 5, 1-17. 